\title{
Estudo da adsorção de dispersante à base de poliacrilato em um sistema varistor comercial: caracterização físico-química
}

\section{(Study of polyacrylate adsorption in a commercial varistor system: characterization of the physical-chemistry properties)}

\author{
S. L. M. Brito ${ }^{1,2}$, D. Gouvêa ${ }^{I}$, R. Ganzella ${ }^{2}$ \\ ${ }^{1}$ Laboratório de Processos Cerâmicos, Departamento de Engenharia Metalúrgica e de Materiais, \\ Escola Politécnica da Universidade de S. Paulo (EPUSP) \\ Av. Prof Mello Moraes, 2463, Cidade Universitária, S. Paulo 05508-900 \\ ${ }^{2}$ Indústria Eletromecânica Balestro Ltda. \\ Rua Santa Cruz, 1550, Mogi Mirim, SP 13800-440 \\ silvio.miranda@poli.usp.br,dgouvea@usp.br
}

\begin{abstract}
Resumo
Um dos grandes desafios no processamento cerâmico é a obtenção de dispersões de partículas estáveis e homogêneas contendo materiais com diferentes características físicas e químicas, como densidade, área de superfície específica, distribuição de tamanho de partículas e ponto isoelétrico. $\mathrm{O}$ comportamento de suspensões obtidas a partir da composição de um sistema varistor à base de $\mathrm{ZnO}$ (aditivos: $\mathrm{Bi}_{2} \mathrm{O}_{3}, \mathrm{Sb}_{2} \mathrm{O}_{3}, \mathrm{Co}_{3} \mathrm{O}_{4}, \mathrm{Mn}_{2} \mathrm{O}_{3}, \mathrm{NiO}, \mathrm{Cr}_{2} \mathrm{O}_{3}$ ) em meio aquoso, utilizando o poliacrilato de amônio como dispersante foi avaliada para determinar a influência dos diferentes óxidos no processamento. Os óxidos foram caracterizados a partir das técnicas mobilidade eletroforética dinâmica, espectroscopia no infravermelho, distribuição granulométrica e área de superfície específica. Os óxidos aditivos apresentam características de superfície, como ponto isoelétricos (pie), distintos com relação ao óxido base (ZnO - pie 9.4). Os dois mais importantes, devido às fortes diferenças de pie foram o $\mathrm{Sb}_{2} \mathrm{O}_{3}$ e o $\mathrm{Cr}_{2} \mathrm{O}_{3}$, pie $=3,4$ e 4,4, respectivamente. Esta diferença de pie entre o $\mathrm{ZnO}$ e os aditivos promovem uma mudança no pH quando em dispersão aquosa, que passa a ser de aproximadamente 8,0 e que pode comprometer a estabilidade da dispersão em algumas etapas do processo de dispersão. Palavras-chave: varistor, $\mathrm{ZnO}$, físico-química de superfície, estabilização eletro-estérica, isoterma de adsorção.
\end{abstract}

\begin{abstract}
One of the greatest challenges of ceramic processing is to obtain a stable and homogeneous dispersion of particles when the components present different physical and chemical characteristics, such as density, specific surface area, size distribution and isoelectric point (iep). The $\mathrm{ZnO}$ varistor composition contains 7 different oxides (additives: $\mathrm{Bi}_{2} \mathrm{O}_{3}, \mathrm{Sb}_{2} \mathrm{O}_{3}, \mathrm{Co}_{3} \mathrm{O}_{4}, \mathrm{Mn}_{2} \mathrm{O}_{3}, \mathrm{NiO}$, and $\mathrm{Cr}_{2} \mathrm{O}_{3}$ ) and the stability of the aqueous dispersion strongly depends on the specific surface area, the iep and the dispersant choice. In this work we evaluated the ammonium polyacrylate $\left(\mathrm{PAA}-\mathrm{NH}_{4}\right)$ as a dispersant to determine the influence of the different oxides on the processing. The oxides have been characterized by electrophoretic mobility, particle size distribution and specific surface area. Some of varistor oxides additives present isoelectric point (iep) distinct from the $\mathrm{ZnO}(\mathrm{iep}=9,4)$. Among them, $\mathrm{Sb}_{2} \mathrm{O}_{3}(\mathrm{iep}=$ $3.4)$ and $\mathrm{Cr}_{2} \mathrm{O}_{3}(\mathrm{iep}=4.4)$ have been considered. This difference between the $\mathrm{ZnO}$ and these additives promotes a change in the $\mathrm{pH}$ dispersion that could compromise the dispersion stability.
\end{abstract}

Keywords: varistor, $\mathrm{ZnO}$, surface physical-chemistry, electrosteric stabilization, isotherm of adsorption.

\section{INTRODUÇÃO}

Os avanços tecnológicos e o reconhecimento das propriedades dos materiais cerâmicos têm feito com que a sua utilização cresça em várias áreas. Propriedades elétricas, por exemplo, fazem com que materiais cerâmicos tenham uma contribuição essencial em funções ativas, como componentes com aplicações eletrônicas. Dentre eles, podese citar alguns componentes cerâmicos com aplicações eletrônicas como: varistores, transdutores piezoeléctricos, capacitores dielétricos, componentes híbridos de filmes finos, ferritas, substratos cerâmicos e vidros; com funções variadas relacionadas ao monitoramento de: temperatura, tensão elétrica, umidade, e presença de gases nocivos[1].

Os varistores de $\mathrm{ZnO}$ são componentes eletro-eletrônicos aplicados à proteção de circuitos. Foram inicialmente desenvolvidos por Matsuoka [2, 3] na década de 60. Apesar de nos dias atuais serem bem conhecidos a influência de aditivos, o processamento térmico, a microestrutura e as características elétricas [4-13], existe uma carência muito 
grande de informações a respeito do processamento e da estabilidade desses materiais em meios aquosos, bem como de técnicas de conformação alternativas.

A aplicação destes componentes está relacionada com sistemas de proteção de sobre-tensão por apresentar característica isolante até um determinado valor de tensão (ou tensão de ruptura) a partir da qual passa a apresentar um comportamento condutor. Assim, quando existe um aumento repentino de tensão acima da tensão de ruptura parte da energia é dissipada na forma de calor protegendo o sistema de possíveis danos [14].

Esses materiais são normalmente prensados em blocos cerâmicos obtidos a partir do material atomizado. A mistura e homogeneização dos diferentes componentes se dão em meio aquoso.

O objetivo deste trabalho é o estudo das propriedades físico-químicas dos óxidos que compõem o sistema varistor, com foco principal em suas características de superfície, avaliando a influência dos mesmos na homogeneidade das dispersões aquosas.

\section{EXPERIMENTAL}

O trabalho foi desenvolvido para uma formulação semelhante à desenvolvida por Matsuoka, porém adaptada pela empresa Balestro, e utilizada na produção de varistores de alta tensão conformados por prensagem para sua linha de produtos pára-raios. A Balestro é a única empresa nacional, fabricante de blocos para pára-raios.

As matérias-primas utilizadas neste trabalho são $\mathrm{ZnO}$, $\mathrm{Bi}_{2} \mathrm{O}_{3}, \mathrm{Sb}_{2} \mathrm{O}_{3}, \mathrm{NiO}, \mathrm{Co}_{3} \mathrm{O}_{4}, \mathrm{Cr}_{2} \mathrm{O}_{3}, \mathrm{Mn}_{2} \mathrm{O}_{3}$, todos de origem internacional, porém algumas das principais informações dos óxidos selecionados pela empresa para atender suas necessidades de processo, qualidade e preço (como fabricante e análise química), além da formulação desenvolvida, não serão descritos neste trabalho, pois como, de comum acordo, devem manter sigilo industrial.

As caracterizações foram feitas utilizando as seguintes técnicas: Análise de Distribuição Granulométrica (Espalhamento por Laser) série 2600 da Malvern Instruments Ltd; Análise de Superfície Específica $\left(\mathrm{S}_{\mathrm{BET}}\right)$ com um Gemini III 2375 Surface Area Analyser da Micromeritics; Espectroscopia de Infravermelho (FT-IR) um Magna 560 com célula DRIFT da Nicolet Instrument Corporation; Mobilidade Eletroforética Dinâmica (ESA) com um ESA 8000 da Matec Applied Sciences.

As análises objetivaram uma avaliação das melhores condições de trabalho, como por exemplo, processamento na mistura, a quantidade mínima de dispersante para promover uma suspensão desaglomerada e com um alto grau de estabilidade, e ainda da possibilidade da adição de aditivos, como plastificantes e/ou ligantes, que algumas técnicas de conformação exigem.

Para as análises titulométricas ( $\mathrm{ZnO}$ e óxidos aditivos) e isoterma de adsorção (Mistura) foram utilizadas suspensões com fração volumétrica de $2 \%$ de material sólido, além de controle de temperatura para $25^{\circ} \mathrm{C}$. Todas análises foram feitas utilizando-se água bidestilada/deionizada, $\mathrm{pH}$ de $5,7 \pm 0,1 \mathrm{e}$ condutividade iônica de $2 \mu \mathrm{S} / \mathrm{cm}$. O dispersante utilizado foi um poliacrilato de amônio comercial.

\section{RESULTADOS E DISCUSSÃO}

\section{Caracterização dos óxidos que compõem o sistema} varistor

O sistema varistor é um sistema complexo que contém uma mistura de vários óxidos. Durante o processo de fabricação é muito importante à garantia da homogeneidade da mistura, já que várias etapas do processamento podem ser afetadas pela distribuição dos aditivos na matriz de $\mathrm{ZnO}$. Desta forma, as informações das características físicoquímicas dos materiais e principalmente de sua superfície, são muito importantes e podem contribuir de forma considerável para que se obtenha uma mistura homogênea. $\mathrm{O}$ cuidado com a mistura dos componentes pode ser importante não só para a qualidade do processamento, como também as características do produto após conformação e sinterização. Contudo, o $\mathrm{ZnO}$, por ser o óxido majoritário neste sistema, deve ser considerado com mais atenção.

A análise granulométrica do $\mathrm{ZnO}$ mostra uma distribuição bastante estreita, como pode ser visto na Fig. 1. Os diâmetros médios foram calculados utilizando médias estatísticas e são apresentados por diâmetro médio Herdan ou De Brouckere - D [4, 3] igual a 1,69 $\mu \mathrm{m}$, e diâmetro médio de Sauter - D [3, 2] igual a 1,68 $\mu \mathrm{m}$. O trabalho com um pó de distribuição estreita pode ser considerado como um fator que facilita o controle da sinterização, contudo diminui o fator de empacotamento, que tem como conseqüência a impossibilidade de se obter dispersões com altas concentrações de sólidos.

A análise da área de superfície específica (ASE), obtida pelo método BET, resultou em $3,66 \mathrm{~m}^{2} / \mathrm{g}$. O valor do tamanho médio de grãos calculado a partir das medidas de

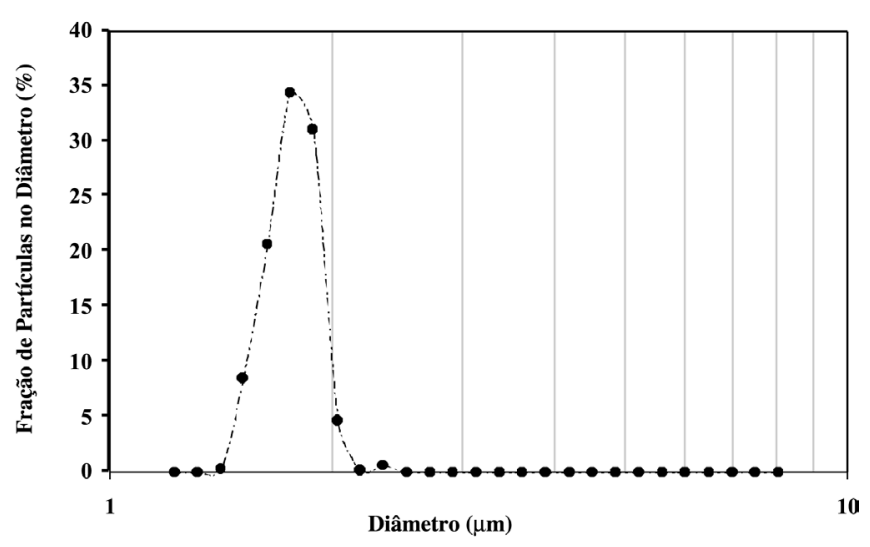

Figura 1: Distribuição granulométrica do $\mathrm{ZnO}$.

[Figure 1: Particle size distribution of $\mathrm{ZnO}$ powder.] 
ASE utilizando a relação ASE $=6 / \rho . d$ [15] é então 0,29 $\mu \mathrm{m}$, onde $\rho$ é a densidade do material e $\mathrm{d}$ o diâmetro médio da partícula. A discrepância entre os valores obtidos a partir das duas técnicas vem do fato que o material pode apresentar um certo grau de aglomeração.

As análises de mobilidade eletrocinética tiveram como objetivo determinar as características de cargas superficiais dos óxidos em função do $\mathrm{pH}$, fator que tem grande influência no comportamento da suspensão. A Fig. 2 mostra o gráfico de potencial zeta em função do $\mathrm{pH}$ para o $\mathrm{ZnO}$. Pode-se observar que o $\mathrm{ZnO}$ tem uma densidade de carga predominantemente positiva quando introduzido em meio aquoso e a suspensão adquire $\mathrm{pH} \cong 7,0$ sem qualquer aditivo.

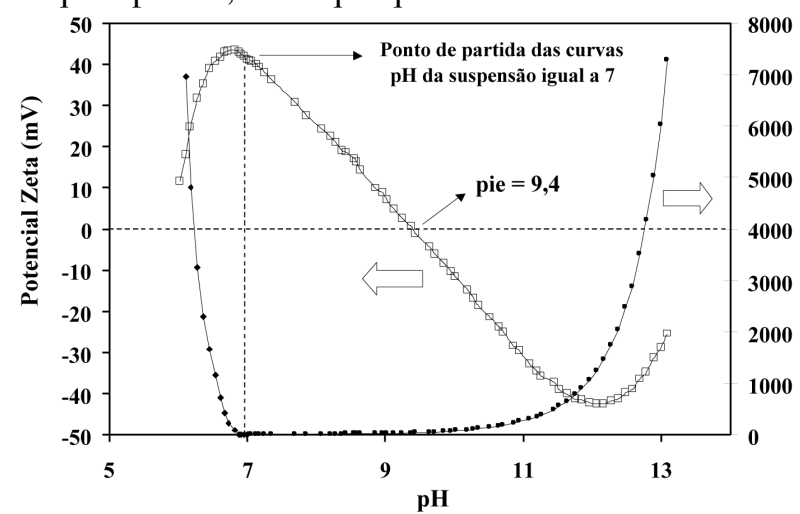

Figura 2: Potencial zeta e condutividade elétrica em função do $\mathrm{pH}$ para o $\mathrm{ZnO}$. (variação de $\mathrm{pH} 7$ à 6 com adição de $\mathrm{HNO}_{3}(2 \mathrm{~N})$ e de pH 7 à 13 com adição de $\mathrm{KOH}(2 \mathrm{~N}))$.

[Figure 2: Zeta potential and the electric conductivity of the dispersion versus $\mathrm{pH}$ for the $\mathrm{ZnO}$ powder (acid variation with $\mathrm{HNO}_{3}(2 \mathrm{~N})$ and basic variation with $\left.\mathrm{KOH}(2 \mathrm{~N})\right)$.]

Desta forma, o sistema já apresenta um potencial zeta elevado e em torno de $40 \mathrm{mV}$, o que provavelmente garantiria a estabilidade da dispersão sem qualquer aditivo. A análise foi então executada em duas etapas, a saber: de $\mathrm{pH} 7$ a 6 com adição de solução de $\mathrm{HNO}_{3}(2 \mathrm{~N})$ e de $\mathrm{pH} 7$ a 13 com adição de solução de $\mathrm{KOH}(2 \mathrm{~N})$. A titulação determinou o valor de 9,4 para o pie. Este comportamento é especial já que para a maioria dos materiais, quando o pó é adicionado em água sem quaisquer aditivos, o pH da dispersão normalmente é próximo do pie.

A presença de grupos carregados na superfície de partículas contribui para um efeito de repulsão ou atração dos polieletrólitos à superfície. A adsorção específica de dispersantes contendo grupos carboxílicos à interface óxido/líquido é ocasionada pela substituição (reação de coordenação) de íons hidroxila $\left(-\mathrm{OH}^{-}\right)$ou grupos $-\mathrm{OH}_{2}^{+}$ presentes na superfície das partículas do óxido pelos íons carboxilato $\left(-\mathrm{COO}^{-}\right)$da molécula do dispersante [16]. É assumido que a substituição de grupos superficiais $-\mathrm{OH}_{2}^{+}$se dê de forma preferencial e que a afinidade dos polieletrólitos aniônicos por partículas apresentando grupos superficiais carregados positivamente é bem maior, porém não podemos dizer que esta afinidade seja nula para partículas apresentando grupos superficiais carregados negativamente.
Sabendo que a adsorção específica de polieletrólitos aniônicos (como é o caso do poliacrilato de amônio) se dá preferencialmente em óxidos com pie básico, pode-se dizer que deve existir a afinidade polímero-partícula neste sistema.

$\mathrm{O}$ espectro de FTIR do $\mathrm{ZnO}$ obtido com uma célula de refletância difusa é mostrado na Fig. 3. Pode-se observar a presença de uma grande quantidade de água adsorvida e não dissociada, hidroxilas, pequenas quantidades de $\mathrm{CO}_{2} \mathrm{e}$ moléculas de espécies orgânicas adsorvidas. A presença de moléculas como $\mathrm{CO}_{2}$ pode explicar o valor de $\mathrm{pH}$ da dispersão muito diferente do pie. De forma geral, quando um óxido é adicionado em água, o pH final da dispersão é próximo ao pie [17-19]. No caso do $\mathrm{ZnO}$, o pH final da dispersão fica em torno de 7. Isto é uma forte evidência de que o $\mathrm{CO}_{2}$ adsorvido na superfície do $\mathrm{ZnO}$ se dissocia em água dando origem ao ácido carbônico, que modifica o $\mathrm{pH}$ da dispersão e gera um forte potencial zeta mesmo sem a introdução de outros aditivos. O espectro de FTIR por DRIFT evidencia hidroxilas a $3.700 \mathrm{~cm}^{-1}$ que são características de materiais com pie básico e que está em acordo com as medidas de potencial zeta em função do $\mathrm{pH}$. A presença de ligações $\mathrm{C}-\mathrm{H}$ $\left(\sim 3.000 \mathrm{~cm}^{-1}\right)$ também é uma evidência de que a superfície do material apresenta impurezas provavelmente do processo de síntese.

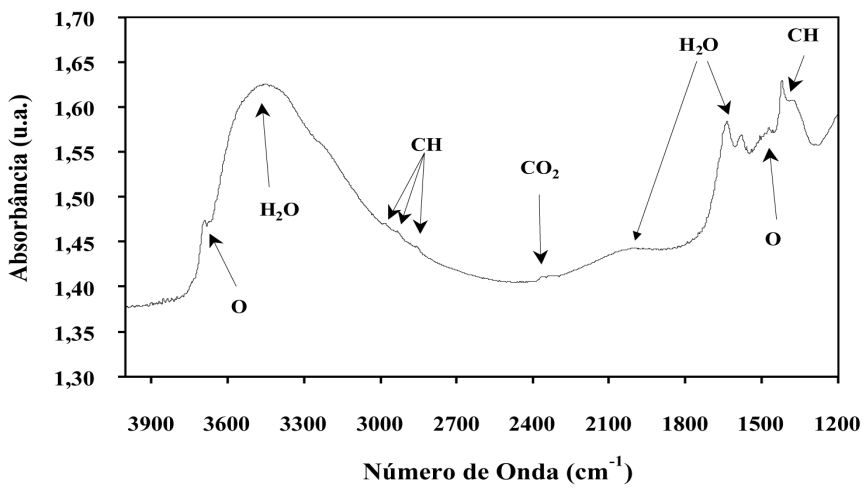

Figura 3: Espectro de FTIR obtido com célula de refletância difusa do $\mathrm{ZnO}$.

[Figure 3: DRIFT of the $\mathrm{ZnO}$ powder.]

Os óxidos aditivos utilizados no sistema varistor foram caracterizados com as mesmas técnicas e as informações mais importantes obtidas são apresentadas na Tabela I e Tabela II.

As análises mostram que alguns óxidos como o $\mathrm{Sb}_{2} \mathrm{O}_{3}$, $\mathrm{Co}_{3} \mathrm{O}_{4}, \mathrm{NiO}$ e o $\mathrm{Cr}_{2} \mathrm{O}_{3}$, têm diâmetros médios relativamente baixos, distribuição estreita e áreas de superfície consideradas não muito baixas para processos cerâmicos, o que deve favorecer a atividade do material no sistema, além de uma distribuição mais homogênea. Na Fig. 4, pode-se observar que o óxido de manganês apresentou um tamanho médio de partículas alto, com distribuição concentrada em duas famílias, porém sua área de superfície foi a mais alta, isto pode ser um indício da existência de aglomerados. 
Tabela I - Distribuição granulométrica e área de superfície específica dos óxidos aditivos do sistema varistor. [Table I - Granulometric distribution and specific surface area of the additives of the varistor formulation.]

\begin{tabular}{cccc}
\hline Material & $\mathrm{D}[3,2](\mu \mathrm{m})$ & $\mathrm{S}_{\mathrm{BET}}\left(\mathrm{m}^{2} / \mathrm{g}\right)$ & Comentários \\
\hline $\mathrm{Bi}_{2} \mathrm{O}_{3}$ & 7,32 & 0,45 & distribuição aberta \\
\hline $\mathrm{Sb}_{2} \mathrm{O}_{3}$ & 3,51 & 3,33 & distribuição relativamente estreita \\
\hline $\mathrm{NiO}$ & 5,26 & 5,32 & distribuição aberta com 3 famílias \\
& & & $(2,5$ e $10 \mu \mathrm{m})$ \\
\hline $\mathrm{Co}_{3} \mathrm{O}_{4}$ & 4,34 & 3,84 & distribuição relativamente estreita \\
\hline $\mathrm{Cr}_{2} \mathrm{O}_{3}$ & 3,14 & 2,31 & distribuição relativamente estreita \\
\hline $\mathrm{Mn}_{2} \mathrm{O}_{3}$ & 11,23 & 6,06 & distribuição aberta com 2 famílias pequenas \\
& & $(3$ e 40 um) e 2 grandes (10 e 20 $\mu \mathrm{m})$
\end{tabular}

Tabela II - Propriedades eletrocinéticas dos óxidos aditivos do sistema varistor. [Table II - Electrokinetic properties of the oxides of the varistor system.]

\begin{tabular}{|c|c|c|c|}
\hline Material & $\begin{array}{c}\text { Faixa de pH com } \\
\text { densidade de carga }+\end{array}$ & $\begin{array}{l}\text { Faixa de pH com } \\
\text { densidade de carga - }\end{array}$ & pie \\
\hline $\mathrm{Bi}_{2} \mathrm{O}_{3}$ & $2-9,7$ & $9,7-13$ & 9,7 \\
\hline $\mathrm{Sb}_{2} \mathrm{O}_{3}$ & $2-3,4$ & $3,4-11$ & 3,4 \\
\hline $\mathrm{NiO}$ & $2-10,3$ & $10,3-13$ & 10,3 \\
\hline $\mathrm{Co}_{3} \mathrm{O}_{4}$ & $2-6,3$ & $6,3-13$ & 6,3 \\
\hline $\mathrm{Cr}_{2} \mathrm{O}_{3}$ & $2-4,4$ & $4,4-12$ & 4,4 \\
\hline $\mathrm{Mn}_{2} \mathrm{O}_{3}$ & $2-5,6$ & $5,6-12,5$ & 5,6 \\
\hline
\end{tabular}

Já o $\mathrm{Bi}_{2} \mathrm{O}_{3}$ apresentou diâmetro médio de partículas relativamente alto, distribuição aberta e área de superfície muito baixa. Estas características podem ser desfavoráveis para sua homogeneidade no sistema e pode ter conseqüências indesejáveis para as propriedades finais, considerando que o óxido de bismuto tem a função de formar fases como pirocloro e espinélio além da fase líquida, promovendo reações e dissolução dos demais dopantes. Trabalhos de moagem neste óxido, devem tornar a mistura mais homogênea, podendo melhorar as características elétricas do componente.

Os resultados de ESA mostraram que o $\mathrm{Sb}_{2} \mathrm{O}_{3}, \mathrm{o}_{2} \mathrm{Cr}_{3}$ e o $\mathrm{Mn}_{2} \mathrm{O}_{3}$ têm seus pontos isoelétricos em $\mathrm{pH}$ ácido, como pode ser visto na Tabela II. Esta característica de superfície pode influenciar diretamente no $\mathrm{pH}$ da suspensão, pois a mistura destes componentes deve agir de modo a reduzir o $\mathrm{pH}$, principalmente no caso do $\mathrm{Sb}_{2} \mathrm{O}_{3}$ onde este efeito deve ser mais crítico, porque este, tem pie extremamente ácido e é o óxido aditivo em maior quantidade. Então alguns cuidados como ajuste de $\mathrm{pH}$ para estes óxidos também podem vir a aumentar o grau de homogeneidade da mistura. Os demais óxidos, assim como o $\mathrm{ZnO}$, têm o ponto isoelétrico básico, característica que não deve influenciar de forma determinante o pH da suspensão quando adicionados.

A Fig. 5 confirma a influência que os óxidos aditivos têm sobre a curva de potencial zeta em função do $\mathrm{pH}$. O ponto isoelétrico passa de $9,4(\mathrm{ZnO})$ para aproximadamente 8 no 


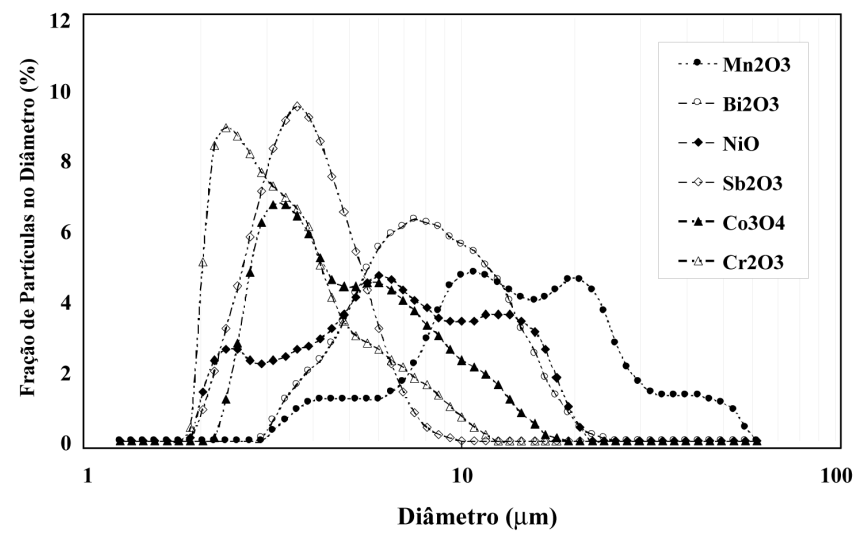

Figura 4: Distribuição granulométrica dos aditivos.

[Figura 4: Particle size distribution of addictive powders.]

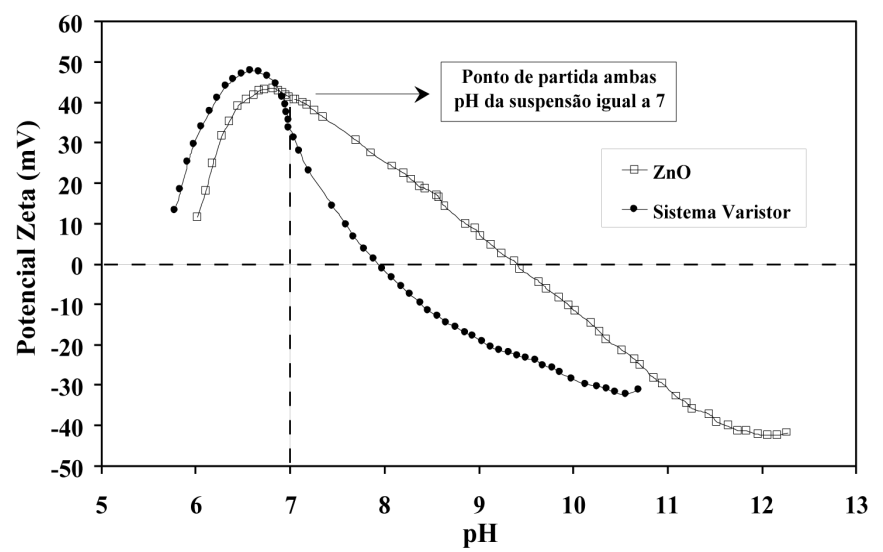

Figura 5: Potencial zeta em função do $\mathrm{pH}$ para a composição do varistor em comparação com os resultados para o $\mathrm{ZnO}$ sem aditivos.

[Figure 5: Zeta potential versus pH obtained by ESA for the varistor compositions and $\mathrm{ZnO}$ powder.]

sistema varistor, caracterizando a mudança de comportamento com a presença de óxidos com características de superfície ácida. Pode-se observar que é possível atingir um certo grau de estabilidade, apenas alterando o $\mathrm{pH}$ da suspensão. Porém nestas faixas de $\mathrm{pH}$, onde se deve obter uma dispersão estável, acontece para valores extremos e a quantidade de base a ser adicionada é relativamente grande fazendo com que a força iônica do meio aumente consideravelmente. Além disto, o controle da estabilidade através do $\mathrm{pH}$ pode apresentar dificuldades oriundas da solubilidade que alguns aditivos e principalmente o $\mathrm{ZnO}$ podem apresentar em $\mathrm{pH}$ muito ácidos ou básicos.

A partir desses fatos considera-se que a melhor forma de geração de cargas para obtenção de uma dispersão com alto grau de estabilidade é através de dispersantes, como polieletrólitos à base de poliacrilato de amônio, a fim de gerar efeitos eletro-estéricos para obtenção de uma dispersão estável e homogênea com elevado potencial zeta e baixa condutividade elétrica.

Grau de dissociação do dispersante versus $\mathrm{pH}$
O poliacrilato de amônio (PAA-NH 4 ), é um polieletrólito formado a partir da reação de neutralização do ácido poliacrílico com hidróxido de amônio $\left(\mathrm{NH}_{4} \mathrm{OH}\right)$ [20]. Pelo fato dos grupos carboxílicos $(\mathrm{COOH})$ sofrerem dissociação em meio aquoso dando origem aos grupos carboxilato $\left(\mathrm{COO}^{-}\right)$(como pode ser visto na equação (A), sendo classificado como polieletrólito aniônico.

$$
\mathrm{RCOOH}+\mathrm{H}_{2} \mathrm{O} \Leftrightarrow \mathrm{RCOO}^{-}+\mathrm{H}_{3} \mathrm{O}^{-}
$$

O grau de dissociação dos grupos carboxílicos presentes ao longo da macromolécula varia em função das condições do solvente como pH e força iônica do meio. Verifica-se na maioria das vezes um aumento do grau de dissociação como decorrência do aumento do $\mathrm{pH}$. Isto ocorre pelo deslocamento do equilíbrio da equação (A) (onde $R$ são unidades repetitivas (meros) das cadeias poliméricas).

Portanto assumindo a reação de dissociação apresentada na equação tem-se uma constante de equilíbrio $K_{1}$ da reação [20], que pode ser escrita da seguinte forma:

$$
\mathrm{K}_{1}=\frac{\left[\mathrm{R}-\mathrm{COO}^{-}\right] \cdot\left[\mathrm{H}_{3} \mathrm{O}^{+}\right]}{[\mathrm{R}-\mathrm{COOH}]}
$$

Esta constante de equilíbrio $K_{1}$ está diretamente relacionada à fração dissociada de grupos funcionais $\left(a_{1}\right)$ conforme descrito pela equação (C)

$$
\mathrm{pK}=-\log \left(\mathrm{K}_{1}\right)=\mathrm{pH}-\log \left(\frac{\alpha_{1}}{1-\alpha_{1}}\right)
$$

Então a determinação do valor de $p K$ pode ser efetuada experimentalmente a partir de uma curva de $\mathrm{pH}$ da solução do polieletrólito em função do volume de ácido adicionado para a variação do $\mathrm{pH}$ desta solução. $\mathrm{O}$ valor de $\mathrm{pH}$ para o qual verifica-se uma inflexão na curva $(\partial p h / \partial V=$ mínimo em módulo ou ainda $\partial p h^{2} / \partial V^{2}$ ) corresponde ao valor de $p K$

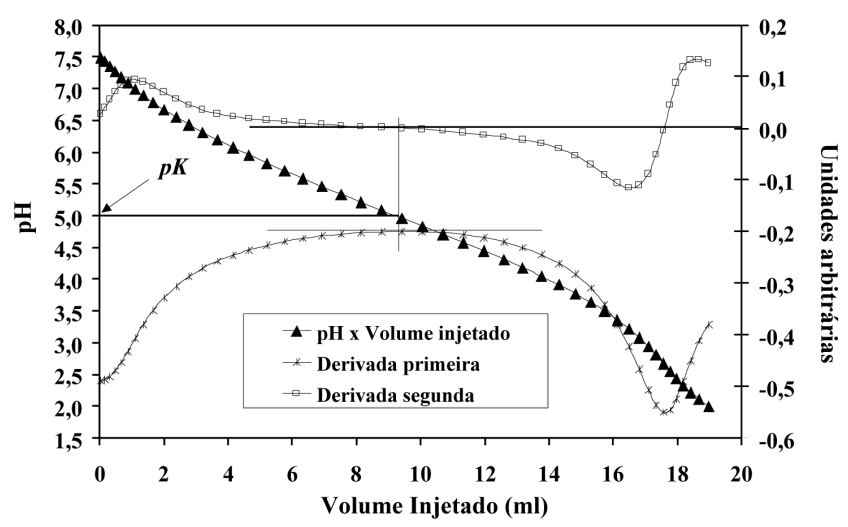

Figura 6: Variação do pH de uma solução aquosa de $\mathrm{PA}^{-\mathrm{NH}_{4}}(1: 20$ em peso) em função da adição de $\mathrm{HNO}_{3}(2 \mathrm{~N})$.

[Figure 6: $\mathrm{pH}$ variation of an aqueous solution of $\mathrm{PAA}-\mathrm{NH}_{4}(1: 20$ wt.) with $\mathrm{HNO}_{3}(2 \mathrm{~N})$ addition.] 
referente ao ponto médio (50\%) dos grupos dissociados.

A Fig. 6 ilustra um experimento feito a partir de uma solução aquosa de poliacrilato de amônio $\mathrm{PA}^{-\mathrm{NH}_{4}}$ (diluição de 1:20) em função da adição de ácido nítrico $\operatorname{HNO}_{3}(2 \mathrm{~N})$. Podemos identificar três regiões distintas neste gráfico. $\mathrm{Na}$ primeira ocorre uma variação de $\mathrm{pH}$ mais significativa com pequenas adições de ácido, gerada pelo aumento da quantidade de íons $\mathrm{H}^{+}$em solução. Na segunda, a variação de $\mathrm{pH}$ é mais suave e sugere que os grupos carboxilato $\left(\mathrm{COO}^{-}\right)$ presentes neste tipo de dispersante estão sendo neutralizados pelos íons $\mathrm{H}^{+}$. Para grandes quantidades de ácido, a variação de $\mathrm{pH}$ volta a ser significativa sugerindo que os grupos carboxilato $\left(\mathrm{COO}^{-}\right)$foram totalmente neutralizados.

Se for analisada a primeira derivada $(\partial p h / \partial v)$ em módulo, podemos observar uma curva apresentando dois picos delimitando um ponto de mínimo. $\mathrm{O}$ valor de $\mathrm{pH}$ deste ponto de mínimo coincide com o valor nulo da segunda derivada $\left(\partial p h^{2} / \partial V^{2}=0\right)$ indicando uma inflexão na curva $p h=f(V)$. $\mathrm{O}$ valor para o qual ocorre este ponto de inflexão equivale ao $p K$.

O valor obtido para $p K$ foi de aproximadamente 5. A equação (D) relaciona o valor de $p K$ e pH com a fração de grupos funcionais dissociados $\left(\alpha_{1}\right)$ [20]

$$
\alpha_{1}=\frac{10(\mathrm{pH}-\mathrm{pK})}{1+10(\mathrm{pH}-\mathrm{pK})}
$$

Aplicando a equação (D) é possível a determinação da fração de grupos funcionais dissociados $\left(\alpha_{1}\right)$, em função de diferentes condições de pH. A Fig. 7 indica que a fração dissociada é praticamente nula para valores inferiores a 3,5. Contudo o aumento do $\mathrm{pH}$ eleva o grau de dissociação, tendo uma fração de grupos funcionais dissociados de praticamente $100 \%$ acima do $\mathrm{pH}$ 7. O grau de estabilidade de uma suspensão, cujo dispersante tenha ácidos carboxílicos como grupos funcionais, fica comprometida para baixos valores

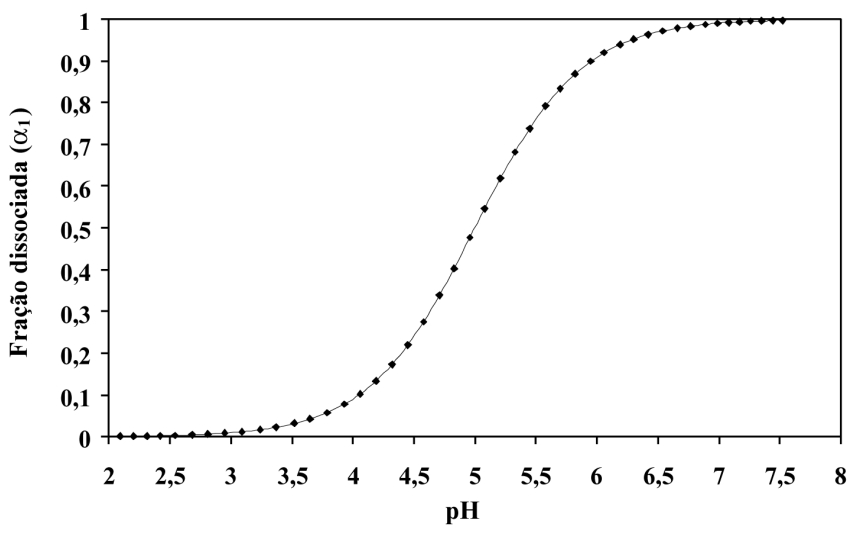

Figura 7: Efeito do $\mathrm{pH}$ sobre a fração de grupos funcionais dissociados para o $\mathrm{PA}-\mathrm{NH}_{4}$.

[Figure 7: Titration of the PA-NH $\mathrm{N}_{4}$ functional groups.] de $\mathrm{pH}$. O dispersante analisado pode não ser totalmente eficiente para valores de $\mathrm{pH}$ da suspensão abaixo de 7 .

Isoterma de Adsorção pelo Método "zeta-sorption”

O método "zeta - sorption" $[18,21]$ propõe a determinação indireta da quantidade de dispersante adsorvida na superfície da partícula $\mathrm{C}_{\mathrm{A}}(\mathrm{mg} / \mathrm{g})$, e este valor pode ser determinado a partir de uma curva de mobilidade eletrocinética ou potencial zeta ( $\zeta)$ em função da quantidade de dispersante (total ativo) adicionado $\mathrm{C}_{\mathrm{L}}(\mathrm{mg} / \mathrm{g})$.

Portanto a curva obtida em análise eletrocinética $\zeta=f$ $\left(\mathrm{C}_{\mathrm{L}}\right)$ é o único dado experimental necessário para aplicação do método, com a ressalva de que existe um intervalo restrito de $\mathrm{pH}$ onde ele é válido, onde é necessário que a variação relativa do $\mathrm{pH}$ não seja significativa com a adição de dispersante $\left(\mathrm{pH}_{\text {normalizado }}\right)$, ou seja, a variação relativa não pode ser maior que $1 \%$, garantindo assim que a geração de cargas na superfície da partícula se deve apenas a adição do dispersante sem efeitos de mudança de $\mathrm{pH}$.

A análise foi feita normalizando o $\mathrm{pH}$, tanto da suspensão quanto da solução de dispersante 1:20, para $\mathrm{pH}=$ 8,7 (adicionando $\mathrm{NH}_{4} \mathrm{OH}-2 \mathrm{~N}$ ). A Fig. 8 mostra uma curva de potencial zeta em função da quantidade de dispersante (total ativo) adicionado (condição de $\mathrm{pH}$ normalizado para 8,7 ), após tratamento matemático dos dados através do método "zeta sorption", onde foi ajustado um polinômio de sexta ordem sobre os pontos experimentais $\left(\mathrm{y}=7,2091 \mathrm{x}^{6}\right.$ $\left.-62,979 x^{5}+221,45 x^{4}-401,33 x^{3}+399,44 x^{2}-217,24 x-14,166\right)$ fazendo uma regressão polinomial com o software Microsoft Excel (Micrososft Corporation Copyright 1983-1997). O intercepto com o eixo y $(\mathrm{x}=0)$ foi adotado como sendo igual ao potencial zeta inicial da análise.

$\mathrm{O}$ método propõe que a reta tangente exatamente no ponto de intercepto com o eixo $y(x=0)$ dada por $y(x)=a_{1} \cdot x+a_{0}$ a eficiência de adsorção é de $100 \%$, então podemos extrair uma relação entre quantidade de dispersante adicionado $\left(\mathrm{C}_{\mathrm{L}}\right)$

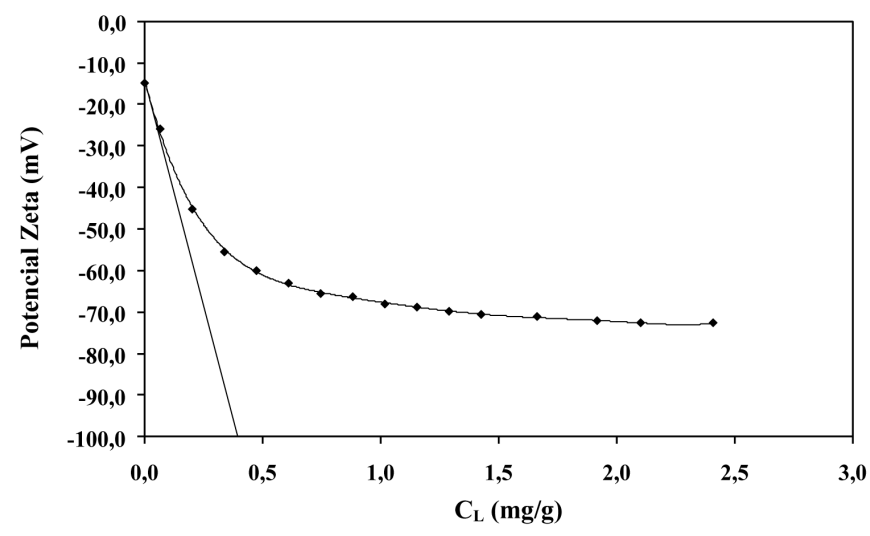

Figura 8: Curva do potencial zeta em função da adição de dispersante após tratamento de dados proposto pelo método "zeta-sorption" ( $\mathrm{pH}$ da suspensão e $\mathrm{pH}$ do dispersante 8,7).

[Figure 8: Zeta potential with the dispersant addition employing the "zeta-sorption" method (suspension and dispersant $\mathrm{pH}$ equal to 8.7$)$.] 


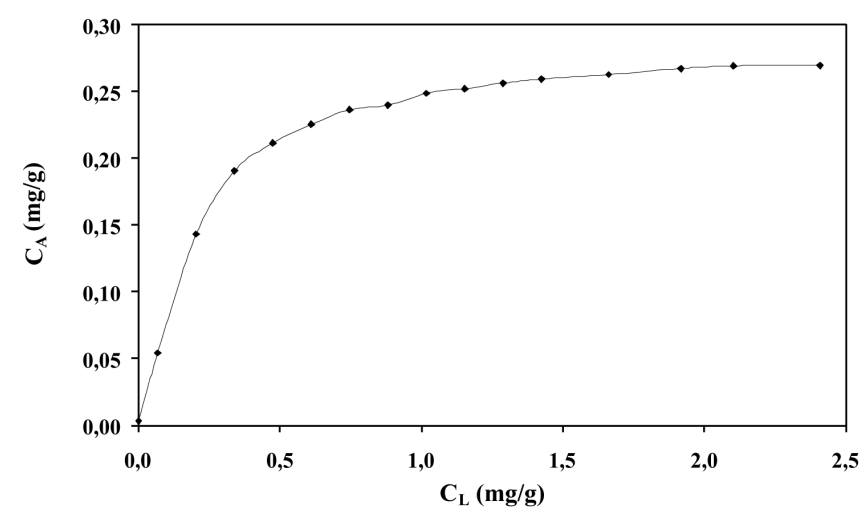

Figura 9: Curva de adsorção do $\mathrm{PAA}-\mathrm{NH}_{4}$ em uma suspensão com $2 \%$ em volume da mistura de pós varistora.

[Figure 9: Adsorption curve of $\mathrm{PAA}-\mathrm{NH}_{4}$ on a 2 vol.\% varistor suspension.]

utilizando o polinônio de sexta ordem ajustado, e a quantidade de dispersante adsorvido $\left(\mathrm{C}_{\mathrm{A}}\right)$ para um mesmo potencial gerado a partir da equação $y=-217,24 x-14,166$. A Fig. 9 mostra a quantidade de dispersante adsorvida em função da quantidade de dispersante adicionada obtida pelo método.

Pode-se observar que ocorre um limite de saturação da superfície, em torno de $\mathrm{C}_{\mathrm{L}}$ igual a $1,5 \mathrm{mg} / \mathrm{g}$, onde a partir deste ponto a maioria do dispersante adicionado fica em solução.

Estes resultados demonstram que as propriedades dos diferentes materiais utilizados no sistema varistor podem ser em alguns momentos do processo fundamentais para a estabilidade da dispersão, porém o $\mathrm{ZnO}$ que é o óxido em maior quantidade determina as características de estabilidade da dispersão.

\section{CONCLUSÕES}

A caracterização dos óxidos que compõem o sistema varistor mostrou que alguns fatores podem comprometer a qualidade do produto, se tratando de homogeneidade da mistura, ou seja, pode-se obter ganho na qualidade final do produto considerando etapas de processamento como por exemplo: moagem dos óxidos aditivos devido à distribuição e tamanho médio de partícula relativamente grandes, como no caso do $\mathrm{Bi}_{2} \mathrm{O}_{3}$; além de ajustes no $\mathrm{pH}$ para controle de carga de superfície dos óxidos com características ácidas, que ao serem introduzidos podem não ficar distribuídos homogeneamente na mistura.

O dispersante (poliacrilato de amônio), utilizado de acordo com as análises de dissociação, tem eficiência de adsorção $100 \%$ para $\mathrm{pH}$ acima de 7 e eficiência praticamente nula para $\mathrm{pH}$ abaixo de 3,5. Em pH 8,7, mostrou-se eficiente e com qualidade gerando alto potencial zeta na mistura, com potencial zeta em torno de $-70 \mathrm{mV}$.

O método "zeta sorption" pôde ser aplicado sem problemas no tratamento da determinação de isotermas de adsorção para $\mathrm{pH}$ normalizado para 8,7, pois não houve variação de $\mathrm{pH}$ da mistura com adição do dispersante.

$\mathrm{O} \mathrm{ZnO}$, como é o material em maior quantidade no sistema, passa a controlar a estabilidade da dispersão.

\section{AGRADECIMENTOS}

Os autores agradecem à FAPESP (Procs. 99/10798-0 e 97/06152-2) e à Indústria Eletromecânica Balestro LTDA.

\section{REFERÊNCIAS}

[1] R. C. Buchanan, Ceramic Materials for Eletronics - Processing, Properties, and Applications, Marcel Dekker Inc, New York, USA (1986) p. iii.

[2] M. Matsuoka, T. Masuyama, Jpn. J. Appl. Phys. 8 (1969) 1275 .

[3] M. Matsuoka, Jpn. J. Appl. Phys. 10, 6 (1971) 736. [4] C. Zhang, D. Zhou, W. Lu, Y. Hu, J. Mater. Sci.: Materials in Eletronics 12 (2001) 357.

[5] S. Bernik, P. Zupancic, D. Kolar, J. Eur. Ceram. Soc. 19 (1999) 709.

[6] A. T. Santhanam, T. K. Gupta, W. G. Carlson, J. Appl. Phys. 50, 2 (1979) 852.

[7] E. Olsson, I. K. L. Falk, G. E. Dunlop, R. Osterlund, J. Mater. Sci. 20 (1985) 4091.

[8] T. Takemura, M. Kobayashi, Y. Takada, K. Sato, J. Am. Ceram. Soc. 70, 4 (1987) 237.

[9] J. Han, P. Q. Mantas, A. M. R. Senos, J. Eur. Ceram. Soc. 20 (2000) 2753.

[10] S. Hindorani, D. O. Shah, M. S. Multani, J. Mater. Res. 10, 2 (1995) 461.

[11] J. P. Gambino, W. D. Kingery, G. E. Pike, L. M. Levinson, H. R. Philipp, J. Am. Ceram. Soc. 72, 4 (1989) 642.

[12] E. Olsson, G. Dunlop, R. Osterlund, J. Am. Ceram. Soc. 76, 1 (1993) 65.

[13] T. Senda, R. Bradt, J. Am. Ceram. Soc. 74, 6 (1991) 1296.

[14] T. K. Gupta, J. Am. Ceram. Soc. 73, 7 (1990) 1817.

[15] T. Allen, Particle Size Measurement, Chapman and Hall, New York, USA (1990).

[16] P. Hidber, T. Graule, L. Gauckler, J. Am. Ceram. Soc. 79, 7 (1996) 1857.

[17] K. L. Guimarães, D. Gouvêa, Applications and Misapplications of The "Zeta-Sorption" Method to Evaluate the Adsorption Behavior of Electrosteric Stabilized Systems, Adv. Colloid Interface Sci. (2004) submetido.

[18] R. H. R. Castro, D. Gouvêa, J. Eur. Ceram. Soc. 23 (2003) 897.

[19] B. B. S. Murad, D. Gouvêa, Cerâmica 45, 292-293 (1999) 103.

[20] J. Cesarano Iii, I. A. Aksay, A. Bleier, J. Am. Ceram. Soc. 71, 4 (1988) 250.

[21] K. L. Guimarães, Aplicação do Método "zeta-sorption" para Avaliação do Efeito do $\mathrm{pH}$ e da Temperatura sobre a Eficiência de Adsorção do Sistema PA- $\mathrm{NH}_{4} / \mathrm{Al}_{2} \mathrm{O}_{3}$, Dissertação de Mestrado, Departamento de Engenharia Metalúrgica e de Materiais, EPUSP (2003).

(Rec. 21/07/2004, Rev. 05/11/2004, Ac. 12/11/2004) 